\title{
Non-geodesic circular motion of massive spinning test bodies around a Schwarzschild field in the Lagrangian theory
}

\author{
Sergio A. Hojman ${ }^{1,2,3,4, a}$, Felipe A. Asenjo ${ }^{5, b}$ \\ ${ }^{1}$ Departamento de Ciencias, Facultad de Artes Liberales, Universidad Adolfo Ibáñez, Santiago, Chile \\ ${ }^{2}$ Centro de Investigación en Matemáticas, A.C, Unidad Mérida, Mérida, Yuc, Mexico \\ ${ }^{3}$ Departamento de Física, Facultad de Ciencias, Universidad de Chile, Santiago, Chile \\ ${ }^{4}$ Centro de Recursos Educativos Avanzados, CREA, Santiago, Chile \\ ${ }^{5}$ Facultad de Ingeniería y Ciencias, Universidad Adolfo Ibáñez, Santiago, Chile
}

Received: 13 July 2018 / Accepted: 12 October 2018 / Published online: 20 October 2018

(C) The Author(s) 2018

\begin{abstract}
Recent interest on studying possible violations of the Equivalence Principle has led to the development of space satellite missions testing it for bodies moving on circular orbits around Earth. This experiment establishes that the validity of the equivalence principle is independent of the composition of bodies. However, the internal degrees of freedom of the bodies (such as spin) were not taken into account. In this work, it is shown exactly that the circular orbit motion of test bodies does present a departure from geodesic motion when spin effects are not negligible. Using a Lagrangian theory for spinning massive bodies, an exact solution for their circular motion is found showing that the non-geodesic behavior manifests through different tangential velocities of the test bodies, depending on the orientation of its spin with respect to the total angular momentum of the satellite. Besides, for circular orbits, spinning test bodies present no tangential acceleration. We estimate the difference of the two possible tangential velocities for the case of circular motion of spinning test bodies orbiting Earth.
\end{abstract}

\section{Introduction}

The equivalence principle (EP) is one of the cornerstones of general relativity. Among all the different possible ways in which it has been stated, one of its simplest form (called its weak form) establishes that all bodies fall with the same acceleration in a given gravitational field [1], implying the equivalence between gravitational and inertial masses. Another precise form to enunciate it is that all bodies moving under the influence of gravitational forces only follow geodesics [1]. The EP only applies in a region of spacetime

\footnotetext{
a e-mail: sergio.hojman@uai.cl

be-mail: felipe.asenjo@uai.cl
}

small enough to neglect the inhomogeneities of gravitational fields [1].

In order to determine experimentally the validity of the EP, some experiments have been carried out recently in different settings [2-7]. They consider the trajectories of massive composed falling bodies, measuring their accelerations, and determining whether (or not) the Eötvös ratio parameter that characterizes the falling is non-zero. The Eötvös parameter $\Delta$ measures the relative difference between accelerations for falling test bodies, and according to the EP, it should vanish.

These experiments run from atomic to celestial scales. For instance, in Ref. [2], ${ }^{87} \mathrm{Rb}$ atoms were studied in a vertical free-falling configuration, where the cluster spin was vertically aligned, pointing either up or down. This experiment determined that $\Delta \sim 10^{-7}$, establishing that the experimental results were not in agreement with any of the considered theoretical models for spin-curvature and spin-torsion couplings developed in Refs. [8-10]. Other experiments testing EP with atoms have been performed in Refs. [3,4]. The kind of experiment performed by the MICROSCOPE satellite (MS) mission [5] is different. The aim of this mission was to measure the forces required to maintain two cylindrical test massive bodies in the same circular orbit around Earth. Bodies with the same and different compositions showed no difference on their trajectory behavior, finding an Eötvös ratio of the order $\Delta \sim 10^{-15}$. The MS mission was focused in determining if the atomic composition of massive bodies can produce any violation of EP, and their findings have a strong indication that it does not. Even tough, recent observations have helped to probe the validity of $\mathrm{EP}$ at galactic scales $[6,7]$.

However, these experiments do not consider any internal degree of freedom of the test bodies, such as spin. In general, it is well-known that spin introduces tidal forces that devi- 
ate any massive free-falling spinning body from a geodesic. Therefore, any spinning massive particle does not follow geodesics [11]. The pioneering works of Mathisson [12,13] and Papapetrou [14] showed that the equations of motion for spinning massive particles are non-geodesic, deriving them as limiting cases of rotating fluids moving in gravitational fields. The Mathisson-Papapetrou equations (MPE) have been used to obtain several exact solutions (see for example Refs. [15-19]). However, the MPE present several problems in the description of spinning massive particles. For example, they are dynamical equations of third order that do not preserve the square root of the Casimir operator of the Poincaré group $P_{\mu} P^{\mu}$ (formed by the momentum $P^{\mu}$ ), among others. These difficulties are analyzed below [20].

Action approaches can be used to describe the dynamics of spinning massive particles from first principles [21]. In this work, we use the Lagrangian theory developed in Refs. [2234], which also allow us to avoid the several issues of MPE. This theory can be derived rigourously from first principles allowing the proper treatment of the crucial lack of parallelism between velocity and momentum, which otherwise cannot be obtained as the canonical momentum cannot be appropriately defined (as in MPE). This Lagrangian theory has been used to study spinning massive particles (tops) in different contexts and gravitational fields [26-34], always finding new effects on the dynamics associated to the nongeodesics motion of tops due to spin-gravity coupling. Moreover, in Ref. [22] was shown that this Lagrangian model for tops matches the experimental conclusions of Ref. [2]. The Lagrangian theory exactly predicts the results of ${ }^{87} \mathrm{Rb}$ atoms experiment [2], rigourously showing that in tops in a vertical free-falling trajectory with spins aligned (with the trajectory), the forces induced by the spin-gravity coupling vanishes, and thereby the top does follow a geodesic [22]. Besides, in Ref. [22], a different and more concrete experimental setting was proposed for tops moving "parabolically" in a non-geodesic orbit, where a measurement could be possibly performed.

This previous success in the agreement of the results which stem from Lagrangian theory for tops with experiment, leads to wonder what experimental settings can be appropriated to measure deviations of from geodesic orbits. It is the purpose of this work to study the circular orbits of tops in a Schwarzschild background using this Lagrangian theory, showing how the spin induces non-geodesic motion of the test bodies. We apply these results to estimate these deviations for a possible circular orbit around Earth. There exist other schemes that allow us to study the motion of tops in gravitational fields, such as post-Newtonian approximation for spinning massive particle [35-40]. In particular, the chaotic motions in post-Newtonian systems of spinning compact binaries were investigated in Refs. [41-50]. Also, circular orbits of tops on a Schwarzschild background have been studied using the MPE approach [51,52], and the chaotic motions of tops in this spacetime background were explored in Refs. [53,54]. However, in here we restrict ourselves to the Lagrangian formalism of Sect. 2, as it allows us to obtain an exact solution for the motion of tops in circular trajectories, without the difficulties introduced by the MPE approach.

\section{Lagrangian theory for tops}

The Lagrangian model for spinning particles consider tops with mass $m$, spin $J$, energy $E$ and total angular momentum $j$. The full theory is developed in Refs. [22-28], and we limit ourselves here to highlight its most relevant results.

\subsection{Equations of motion}

It is well-known that the velocity $u^{\mu}$ of a spinning particle is not parallel, in general, to the canonical momentum vector $P^{\mu}$. The velocity vector may, under some circumstances, become spacelike [23-25]. However, the momentum vector remains always timelike and gives rise to the dynamical conservation law of mass $m^{2} \equiv P^{\mu} P_{\mu}>0[24,28]$. The spin of tops is defined through an antisymmetric tensor $S^{\mu \nu}$ (see below). The action $S=\int L d \lambda$ associated to the Lagrangian theory for tops is $\lambda$-reparametrization invariant, where the Lagrangian $L\left(a_{1}, a_{2}, a_{3}, a_{4}\right)=\left(a_{1}\right)^{1 / 2} \mathcal{L}\left(a_{2} / a_{1}, a_{3} /\left(a_{1}\right)^{2}\right.$, $\left.a_{4} /\left(a_{1}\right)^{2}\right)$ is an arbitrary function of four invariants $a_{1}, a_{2}, a_{3}$, $a_{4}$, and $\mathcal{L}$ is an arbitrary function of three variables where $a_{1} \equiv u^{\mu} u_{\mu}, a_{2} \equiv \sigma^{\mu \nu} \sigma_{\mu \nu}=-\operatorname{tr}\left(\sigma^{2}\right), a_{3} \equiv u_{\alpha} \sigma^{\alpha \beta} \sigma_{\beta \gamma} u^{\gamma}$, $a_{4} \equiv \operatorname{det}(\sigma)[22,24,28]$, where $u^{\mu}$ and $\sigma^{\mu \nu}$ are the top's velocity and angular velocity respectively defined in terms of derivatives with respect to the arbitrary parameter $\lambda$ (see Refs. [22-28]). The momentum vector $P_{\mu}$ and the antisymmetric spin tensor $S_{\mu \nu}$ are canonically conjugated to the position and orientation of the top, $P_{\mu} \equiv \partial L / \partial u^{\mu}$ and $S_{\mu \nu} \equiv \partial L / \partial \sigma^{\mu \nu}=-S_{\nu \mu}$. Explicit examples of such Lagrangians can be found in Refs. [23,28]. In this way, it is found that the dynamics of a top describes a non-geodesic behavior, seen through the momentum equation $[24,27,28]$

$$
\frac{D P^{\mu}}{D \lambda} \equiv \dot{P}^{\mu}+\Gamma_{\alpha \beta}^{\mu} P^{\alpha} u^{\beta}=-\frac{1}{2} R^{\mu}{ }_{\nu \alpha \beta} u^{\nu} S^{\alpha \beta},
$$

and the equation for the spin tensor

$$
\frac{D S^{\mu \nu}}{D \lambda} \equiv \dot{S}^{\mu v}+\Gamma_{\alpha \beta}^{\mu} S^{\alpha v} u^{\beta}+\Gamma_{\alpha \beta}^{v} S^{\mu \alpha} u^{\beta}=P^{\mu} u^{v}-u^{\mu} P^{v} .
$$

The overdot represents the derivative with respect to an arbitrary parameter $(\lambda)$, in such a way that velocity $u^{\mu}=\dot{x}^{\mu}$ is the derivative of coordinates. In addition, $\Gamma^{\nu}{ }_{\rho \tau}$ are the 
Christoffel symbols for the metric field $g_{\mu \nu}$ (the speed of light is set equal to 1 ). The six independent components of the antisymmetric spin tensor generate Lorentz transformations, and in order to restrict them to generate three dimensional rotations we impose the Tulczyjew constraint $S^{\mu v} P_{v}=0$ $[20,23,24,55]$. This constraint has been shown to be important in the consistency of a theory for spinning massive particles [20], as it can be deduced as a constraint which emerges from the Lagrangian of the theory, and not an external imposition on the top dynamics [23] (there are other Hamiltonian formulations which do not require these constraints [56]). Lastly, in this theory, the (square) top spin $J^{2} \equiv \frac{1}{2} S^{\mu v} S_{\mu \nu}$ can be shown to be a conserved quantity [24-28].

The non-geodesic behavior of a top moving on a background gravitational field is determined by Eqs. (1) and (2), plus the constraint. As a result, the top can be interpreted as an extended object that is subject to tidal forces due to gravity. Spin gives internal structure to the classical massive particles, and they cannot be longer described as pointlike objects. Due to the fact that any extended object is crossed by infinitely many geodesics (only a pointlike object is traversed by just one geodesic) the averaged motion does not align with any of the constituent geodesics, and the motion is, in general, non-geodesic. Similar effects have been studied for fields [57,58] (which are naturally extended objects) and electromagnetic waves $[57,59,60]$. Thus, one should expect that the inclusion of spin in the dynamics of massive particles should lead to non-geodesic orbits.

\subsection{Comparison with Mathisson-Papapetrou theory}

The above theory does not coincide with the MPE for massive spinning particles [12-14]. As it is discussed in Ref. [20], the MPE present several problems in their description for tops. The MPE formalism is composed by the third-order dynamical equations

$$
\begin{aligned}
& \frac{D P^{\mu}}{D s}=-\frac{1}{2} R_{\nu \alpha \beta}^{\mu} u^{\nu} S^{\alpha \beta}, \\
& P^{\mu}=m u^{\mu}+u_{\nu} \frac{D S^{\mu \nu}}{D s}, \\
& \frac{D S^{\mu \nu}}{D s}+u^{\mu} u_{\alpha} \frac{D S^{\nu \alpha}}{D s}-u^{\nu} u_{\alpha} \frac{D S^{\mu \alpha}}{D s}=0 .
\end{aligned}
$$

where $P^{\mu}$ is the momentum defined in that theory, and $D / D s$ is the $s$-parametrized covariant derivative for the proper time $s$ of the spinning particle. It is not difficult to obtain [20] that the system preserves the timelike behavior of the velocity $u_{\mu} u^{\mu}=1$. However, the MPE establishes that there is no dynamical conservation law for the mass [20]. In fact, it can be proved that the MPE, under the Pirani constraint $S^{\mu v} u_{v}=0$, the square of momentum $P_{\mu} P^{\mu}$ is not a constant of motion [20]. On the other hand, under the Tulczyjew constraint, it can be shown that the MPE implies that the mass is not a constant of motion [20]. Both results of the MPE represent a very undesired behavior for a relativistic theory of massive particles. We refer the reader to Ref. [20], where a deep discussion on the difficulties of the MPE formalism is presented.

Without a Lagrangian formulation the canonical momentum and the spin tensor cannot be appropriately defined. That is the reason the MPE equations have those difficulties, whereas the above Lagrangian theory does not.

\subsection{Integrability and non-integrability in Schwarzschild background}

Several authors have discussed the integrability and nonintegrability of different models for spinning objects in a Schwarzschild field background. For example, the nonintegrability of system formed by a Schwarzschild black hole orbited by a spinning companion in the MathissonPapapetrou formalism under the extreme-mass-ratio limit was proved in Refs. [53,54], showing the chaoticity of the model. Furthermore, in Refs. $[43,61]$ was shown that the post-Newtonian Lagrangian formulation of the two-black hole system with one body spinning can be chaotic. Also, the post-Newtonian Hamiltonian formulation of the two-black hole system with one body spinning was shown to be integrable and regular $[62,63]$. Through the relation between Lagrangian and Hamiltonian approaches at the same postNewtonian order $[64,65]$, it has been proved that these two models are integrable [66].

Differently, the Lagrangian model used along this work and described in Sect. 2.1, allow us to integrate a particular solution, under assumptions detailed in the following section. In general in this model, the spinning particle moving on a gravitational background has, effectively, six degrees of freedom, after taking into account the Tulczyjew constraint $S^{\mu v} P_{v}=0$. Therefore, six constants of motion in involution are needed for the system to be integrable in Liouville's sense. The constants of motion for the spinning particle trajectory as described by the Lagrangian formalism are the rest mass $m^{2}=P^{\mu} P_{\mu}$, the spin $J^{2}=S^{\mu \nu} S_{\mu \nu}$ and one constant $K_{\xi}=P^{\mu} \xi_{\mu}-(1 / 2) S^{\mu \nu} \xi_{\mu ; \nu}$ associated to each of the Killing vectors $\xi^{\mu}$ of the background metric $g_{\mu \nu}$.

In the case of a Schwarzschild background, there are four different Killing vectors. One of them is associated to time translation and other three are associated to three dimensional rotations. Nevertheless, only two combinations of the three components of the conserved angular momentum three dimensional vector are in involution, namely $j^{2} \equiv j_{x}{ }^{2}+j_{y}{ }^{2}+j_{z}{ }^{2}$ and $j_{z}$. Therefore, on a Schwarzschild background, the problem has six constants of motion, five of them in involution. 
In order to be able to integrate the system, we study the simpler case described in the following section, in which we consider an equatorial orbit with the three dimensional spin vector perpendicular to the plane of the orbit. This constitutes a particular exact solution to the system.

\section{Circular motion solution in a Schwarzschild background}

Several different general and exact solutions of the Lagrangian theory for tops have been found in Refs. [22,24-32]. Here we present only the key steps to obtain the solution for a circular motion of the top with spin perpendicular to the plane of motion. We refer to readers to those references for a full and detailed procedure to get the solutions for the equations of motion derived from the Lagrangian theory.

Assuming a Schwarzschild field background (describing approximately the Earth gravitational field), the equatorial motion of a top can be solved exactly, as any equatorial plane can be defined for circular motion to take place. We write the metric in spherical coordinates $g_{t t}=1-2 r_{0} / r$, $g_{r r}=-\left(1-2 r_{0} / r\right)^{-1}, g_{\theta \theta}=-r^{2}, g_{\phi \phi}=-r^{2} \sin ^{2} \theta$, where $r_{0}=G M$ with the gravitational constant $G$ and the Earth mass $M$. The circular motion is defined as such by $\dot{r}=0$. Besides, without any loss of generality, we can study the motion in the plane defined by $\theta=\pi / 2$. If the top is initially in that plane and $\dot{\theta}=0$, then it remains in that equatorial plane, where $P^{\theta}=0[22,24,28]$. In this solution, spin can be chosen to be orthogonal to the equatorial plane $S^{r \theta}=S^{\theta \phi}=S^{0 \theta}=0[22,24,28]$, being parallel or antiparallel to the total angular momentum of the top along the whole trajectory. This total momentum angular $j$ for the top in this trajectory is a conserved quantity. In Refs. $[22,24,28]$ is shown that the general solutions for the momenta equations (1) are $P_{\phi}=(-j \pm E J / m) /(1-\eta)$, and $P_{t}=\left[E \mp j J r_{0} /\left(m r^{3}\right)\right] /(1-\eta)$, with the dimensionless parameter $\eta=J^{2} r_{0} /\left(m^{2} r^{3}\right)$. Here, the \pm stands for two trajectories that depend on the spin orientation, parallel or antiparallel to the total angular momentum of the top, both of them remaining perpendicular to the plane of motion. These two momenta are conserved $\left(\dot{P}_{t}=0\right.$ and $\left.\dot{P}_{\phi}=0\right)$ for circular motion [22,28], and thus, the Eötvös ratio is meaningless for this particular orbit. The non-geodesic motion manifests itself in changes of the velocity, not acceleration.

From the constant of motion $P_{\mu} P^{\mu}=m^{2}$, we get that $P^{r}=0=\left[P_{t}^{2}-\left(P_{\phi}^{2} / r^{2}+m^{2}\right)\left(1-2 r_{0} / r\right)\right]^{1 / 2}$, in consistency with the circular motion solution, and the relation between the radial momentum and the radial velocity $\dot{r}=\left(1-2 r_{0} / r\right)\left(P^{r} / P_{t}\right)=0$, given by solutions of the Lagrangian theory for tops $[22,24,28]$. This constraint deter- mines the energy of each trajectory

$$
\begin{aligned}
\left(E_{ \pm} \mp \frac{j J r_{0}}{m r^{3}}\right)^{2}= & \left(1-\frac{2 r_{0}}{r}\right) \\
& \times\left[m^{2}(1-\eta)^{2}+\frac{1}{r^{2}}\left(-j \pm \frac{E_{ \pm} J}{m}\right)^{2}\right],
\end{aligned}
$$

of a top moving on a circular orbit of radius $r$. The solutions of Eq. (4) for energy can be readily obtained as

$E_{ \pm}=\frac{ \pm \Lambda_{2}+\sqrt{\Lambda_{2}^{2}+\Lambda_{1} \Lambda_{3}}}{\Lambda_{1}}$,

where

$$
\begin{aligned}
& \Lambda_{1}=1-\frac{J^{2}}{m^{2} r^{2}}\left(1-\frac{2 r_{0}}{r}\right), \\
& \Lambda_{2}=\frac{j J}{m r^{2}}\left(\frac{3 r_{0}}{r}-1\right), \\
& \Lambda_{3}=\left(1-\frac{2 r_{0}}{r}\right)\left(\frac{j^{2}}{r^{2}}+m^{2}(1-\eta)^{2}\right)-\frac{j^{2} J^{2} r_{0}^{2}}{m^{2} r^{6}} .
\end{aligned}
$$

Circular orbits are obtained by the study of the behavior of the effective potential $V_{ \pm}(r)=E_{ \pm}(r)^{2}$ [67]. By calculating the zeros of the derivative of the effective potential a condition for the radius of the orbits can be obtained, while their stability can be studied through the positive behavior of its second derivative. If spin is neglected $\left(\Lambda_{1}=1\right.$ and $\Lambda_{2}=0$ ), the effective potential becomes simply $V=$ $\left(1-2 r_{0} / r\right)\left(j^{2} / r^{2}+m^{2}(1-\eta)^{2}\right)$, and from the zeros of the its derivative we obtain a relation between the allowed radius and the angular momentum of spinless particles [67]

$j=\frac{m r \sqrt{r_{0}}}{\sqrt{r-3 r_{0}}}$.

From where we obtain that circular orbits are allowed only for $r>3 r_{0}$. By checking the second derivative of the effective potential, the stability of circular orbits are restricted only to $r>6 r_{0}$. When spin is included, the condition for circular orbits can be obtained by the zeros of the first derivative of the complete solution for energies (5). The calculation is not straightforward, however for a small spin contribution $J \ll$ $j$, and for tops far from the black hole $r_{0} \ll r$, the allowed stable circular orbits are still for $r \gg 6 r_{0}$. This is the case for the estimations of the trajectory deviations for tops around Earth studied in next section. A numerical calculation of the radius of the innermost stable circular orbit from the energies (5) is shown in Fig. 1. We plot the dimensionless distance $z=r / r_{0}$ in terms of the normalized spin $\mathcal{J}=J /\left(m r_{0}\right)$. The behavior of the radius of the innermost stable orbit is shown for $\mathcal{J} \ll 1$, which is consistent with the condition $J \ll j$. For this case, we have that the innermost radius has a linear 


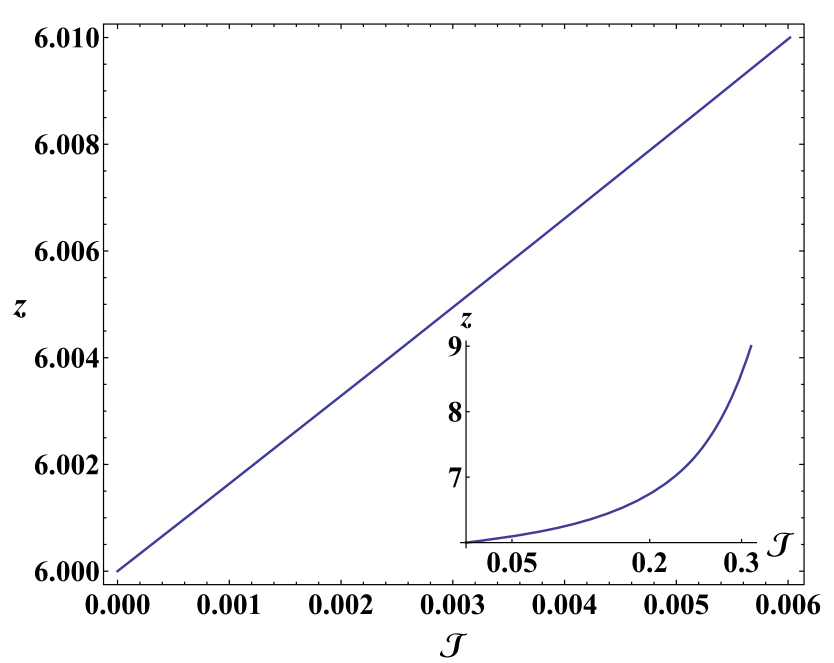

Fig. 1 Radius of the innermost stable circular orbit for non-zero spin contribution from the total energy (5). We plot the dimensionless distance $z=r / r_{0}$ as a function of normalized spin $\mathcal{J}=J /\left(m r_{0}\right)$. We focus in the $\mathcal{J} \ll 1$ regime

relation with spin, with the form of $z>6+\sqrt{4 / 3} \mathcal{J}$. For completeness, in the inset plot we show the behavior of the innermost radius as $\mathcal{J}$ increases. However, this last regime will not be used in the estimations performed in the following section. We can see that our assumptions for stable circular orbits at $r \gg 6 r_{0}$ are justified for small spin contribution.

On the other hand, the non-trivial spin evolution equations (2) relevant to the circular motion in the plane $\theta=\pi / 2$, reduce to $D S^{t r} / D \lambda=0$ and $D S^{t \phi} / D \lambda=P^{t} \dot{\phi}-P^{\phi}[22$, 24,28]. These equations, together with the relations $S^{t r}=$ $-S^{\phi r} P_{\phi} / P_{t}$ and $\left(S^{\phi r}\right)^{2}=J^{2}\left(P_{t}\right)^{2} /\left(m^{2} r^{2}\right)$ that can derived from the two constants of motion and the Tulczyjew condition $[22,24,28]$, allow us to get the angular velocity $\dot{\phi}_{ \pm}$for the two possible trajectories of this motion $[22,24,28]$

$\dot{\phi}_{ \pm}=\frac{1}{r^{2}}\left(1-\frac{2 r_{0}}{r}\right)\left(\frac{2 \eta+1}{\eta-1}\right)\left(\frac{-j \pm E_{ \pm} J / m}{E_{ \pm} \mp j J r_{0} /\left(m r^{3}\right)}\right)$,

where the energy $E_{ \pm}$are given by solutions of (4). Tops can have two tangential velocities $r \dot{\phi}_{ \pm}$, according to Eq. (8). The interplay of its spin with gravity, introduces different corrections in this tangential velocity, which depends on the spin orientation, such that the top with antiparallel spin is faster than the one with the parallel spin to the total angular momentum $r \dot{\phi}_{-}>r \dot{\phi}_{+}$.

A possible measurement of a maximal manifestation of non-geodesic motion can be achieved if two test bodies (with equal composition) are set to rotate in order to have opposite (internal) angular momenta directions, parallel and antiparallel to the total angular momentum of the circular motion of the satellite. In such cases, any deviation from geodesic orbits must be reflected in different measurements of the angular velocities of the test bodies. Thus, the dimensionless ratio

$$
\begin{aligned}
\delta & =\frac{\dot{\phi}_{-}-\dot{\phi}_{+}}{\dot{\phi}_{-}+\dot{\phi}_{+}} \\
& =\frac{\left[-j+\frac{E_{+} J}{m}\right]\left[E_{-}+\frac{j J r_{0}}{m r^{3}}\right]+\left[j+\frac{E_{-} J}{m}\right]\left[E_{+}-\frac{j J r_{0}}{m r^{3}}\right]}{\left[j-\frac{E_{+} J}{m}\right]\left[E_{-}+\frac{j J r_{0}}{m r^{3}}\right]+\left[j+\frac{E_{-} J}{m}\right]\left[E_{+}-\frac{j J r_{0}}{m r^{3}}\right]},
\end{aligned}
$$

is non-zero in the case of circular orbit for the non-geodesic behavior of tops.

Notice that for these circular motions described by solution (8), tops do not present tangential accelerations $\ddot{\phi}=0$, as the radius remains constant. Therefore, there is no relative acceleration between the two test bodies. Furthermore, when spin is neglected $J=0(\eta=0)$, a massive particle can only have a unique angular velocity $\dot{\phi}=j /\left(r^{2} E\right)$ and an unique energy, yielding the usual result $\delta=0$ for geodesic motion in the Schwarzschild field [68]. The approximately vanishing Eötvös ratio and $\delta=0$ are the results measured in the MS mission [5], which is in agreement with the Lagrangian theory for tops.

\section{Estimations of the trajectory deviations}

The inclusion of spin into the test bodies is essential for experiments carried out to demonstrate the validity of nongeodesic motion of massive spinning bodies. To study the first order corrections to the circular orbit of test bodies due to its spin, let us consider the following approximations. Let us study tops motion with $J \ll j$. In this case, the total momentum angular $j$ is approximately by the orbital momentum angular of the top.

At first order in spin, the energy solutions of Eq. (4) is

$$
E_{ \pm} \approx m \sqrt{\left(1-\frac{2 r_{0}}{r}\right)\left(1+\frac{j^{2}}{m^{2} r^{2}}\right)} \mp \frac{j J}{m r^{2}}\left(1-\frac{3 r_{0}}{r}\right)
$$

With this solution, we can obtain the angular velocity (8) of tops at first order in spin to be

$$
\dot{\phi}_{ \pm} \approx \frac{1}{m r^{2}} \sqrt{\frac{1-\frac{2 r_{0}}{r}}{1+\frac{j^{2}}{m^{2} r^{2}}}}\left[j \mp J \sqrt{\frac{1-\frac{2 r_{0}}{r}}{1+\frac{j^{2}}{m^{2} r^{2}}}}\right] .
$$

Therefore, at first order in spin, the $\delta$-parameter (9) becomes

$\delta \approx \frac{J}{j} \sqrt{\frac{1-\frac{2 r_{0}}{r}}{1+\frac{j^{2}}{m^{2} r^{2}}}}$. 
Now, consider a possible experiment near the Earth surface (where $r_{0} \sim 4.4 \times 10^{-3}[\mathrm{~m}] \ll r \sim 7 \times 10^{6}[\mathrm{~m}]$ ) in order to measure the non-geodesic behavior of a top in circular trajectory. This kind of experiment have been performed by the MS mission [5]. Let us consider a model of two test bodies with the same total angular momentum (which is a conserved quantity for each one) but different spin orientations. Thus, considering a small spin contribution and $r_{0} \ll r$, the top total angular momentum can be approximated by Eq. (7). Notice that this angular momentum is consistent with Kepler's law of motion, as for a general angular momentum $j=m r v$ (with test top's velocity $v$ much smaller than the speed of light) we can write $v=r \Omega$ for a circular orbit, where $\Omega$ is the angular frequency of the satellite's trajectory. By Kepler's law, this frequency is related to the trajectory radius by $\Omega^{2}=r_{0} / r^{3}$. Using this, the angular momentum becomes $j \approx m \sqrt{r r_{0}}$, which is an approximation to Eq. (7).

Thus, the energy of top (10) at first order becomes

$E_{ \pm} \approx m\left(1-\frac{r_{0}}{2 r}\right) \mp \frac{J}{r} \sqrt{\frac{r_{0}}{r}}\left(1-\frac{3 r_{0}}{2 r}\right)$

while the approximated angular velocity (11) at first order is

$\dot{\phi}_{ \pm} \approx \frac{1}{r} \sqrt{\frac{r_{0}}{r}}\left(1-\frac{3 r_{0}}{2 r}\right)\left[1+\frac{3 r_{0}}{2 r} \mp \frac{J}{m \sqrt{r_{0} r}}\left(1-\frac{3 r_{0}}{2 r}\right)\right]$.

Notice that the gravitational correction factor $3 r_{0} / 2 r$ to the spin-coupling also appears in post-Newtonian theories [69]. Lastly, the parameter (12) at first order becomes simply

$\delta \approx \frac{J}{m \sqrt{r_{0} r}}\left(1-\frac{3 r_{0}}{r}\right)$.

The behavior of this parameter is shown in Fig. 2, in terms of dimensionless quantities. We have plotted $\delta / \mathcal{J}$ as function of the normalized radius $z=r / r_{0}$ and $\mathcal{J}=J /\left(m r_{0}\right)$. The plot is presented for $z \gg 1$, according to the used approximations. It is straightforward to show from (15) that $\delta / \mathcal{J}=(1-$ $3 / z) / \sqrt{z}$, implying that the parameter has the same behavior for any given spin, depending only on the orbit radius $r$. From the plot, we can see that $\delta$ decays as $1 / \sqrt{z}$.

We can estimate in a closer form the parameter (15) for a test spinning body. Assuming that the intrinsic spin of each top can be estimated as $J \sim m d^{2} \omega$, where $d$ is a characteristic length of the experimental test top body and $\omega$ is its internal angular frequency of rotation. Thus, the correction to the circular trajectory of two massive test objects with different spin orientations can be estimated through the ratio (15) to be $\delta \approx\left(d^{2} \omega / \sqrt{r_{0} r}\right)\left(1-3 r_{0} / r\right)$. This approximated $\delta$-ratio depends on the test top parameters $r, \omega$ and $d$. For motion in a stable circular orbit near the Earth surface $z=r / r_{0} \sim 10^{9}$.

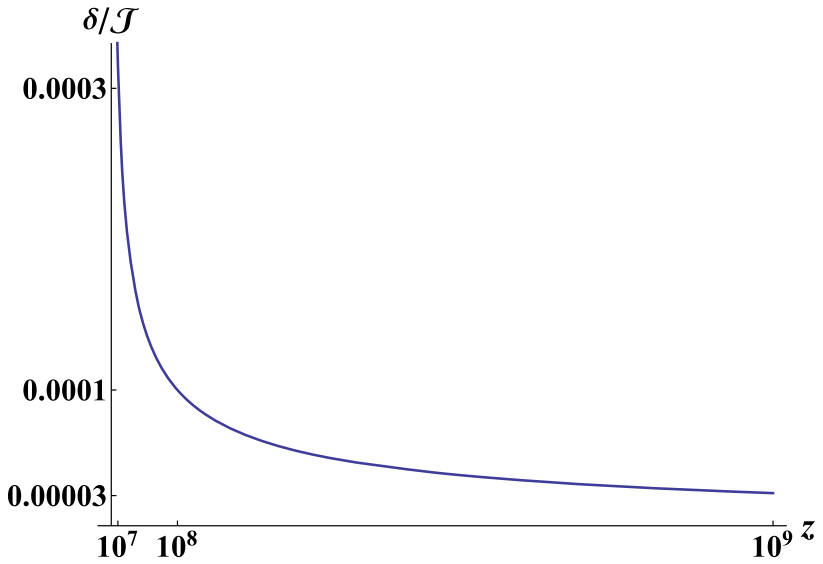

Fig. 2 Behavior of the parameter (15), in terms of $z=r / r_{0}$ and $\mathcal{J}=$ $J /\left(m r_{0}\right)$. The plots are for $z \gg 1$, according to our approximations

Hence, assuming that the test tops have dimensions of the order of $d \sim 10^{-2}[\mathrm{~m}]$, then the ratio gives of the order

$\delta \approx 10^{-15} \omega$.

where the internal angular frequency of tops is measured in $[\mathrm{Hz}]$. For a higher intrinsic angular velocity, a larger deviation from a geodesic path can be achieved.

\section{Conclusions}

The main purpose of this work is to bring attention to the notion of non-geodesic motion, showing that it is the spin (internal dynamics) and not the composition, size or shape of the test bodies, the key for detecting a possible non-geodesic trajectory.

In the simplest case of a circular motion for a spinning massive particle, the complete dynamics can be solved exactly, and it describes a non-geodesics motion whose deviations depend on the magnitude and direction of the particle's spin. Notice that the solution presented above has constant momenta, and thus, the non-geodesic motion cannot be detected by measuring the Eötvös ratio, as the non-geodesic motion is manifested only in the change of the velocities of test bodies. Of course, this is not a general rule, and more complicated motions in different spacetimes may indeed present non-vanishing Eötvös ratios [22,28,30]. As the $\delta$ ratio show in Eq. (9), when spin is negligible then geodesic motion is expected. Therefore, it comes as no surprise that the EP is repeatedly confirmed for non-spinning test bodies.

Any experimental setup designed to measured nongeodesic motion must be constructed in order to capture the spin-gravity coupling and its effects. In particular, for experiments around Earth the $\delta$-ratio has enough freedom to adjust the parameters of the orbit radius of the satellite and the 
characteristic length and inner angular velocity of test body, depending on the accuracy of the setup. Thus, any experimental setting should also consider the angular momenta of test bodies in order to prove the validity of non-geodesic motion due to spin.

Open Access This article is distributed under the terms of the Creative Commons Attribution 4.0 International License (http://creativecomm ons.org/licenses/by/4.0/), which permits unrestricted use, distribution, and reproduction in any medium, provided you give appropriate credit to the original author(s) and the source, provide a link to the Creative Commons license, and indicate if changes were made. Funded by SCOAP ${ }^{3}$.

\section{References}

1. S. Weinberg, Gravitation and Cosmology: Principles and Applications of the General Theory of Relativity (Wiley, New York, 1972)

2. X.-C. Duan et al., Phys. Rev. Lett. 117, 023001 (2016)

3. B.J. Verena, P.K. Majumder, S.K. Lamoreaux, B.R. Heckel, E.N. Fortson, Phys. Rev. Lett. 68, 135 (1992)

4. M.G. Tarallo, T. Mazzoni, N. Poli, D.V. Sutyrin, X. Zhang, G.M. Tino, Phys. Rev. Lett. 113, 023005 (2014)

5. P. Touboul et al., Phys. Rev. Lett. 119, 231101 (2017)

6. L. Shao, N. Wex, M. Kramer, Phys. Rev. Lett. 120, 241104 (2018)

7. A.M. Archibald et al., Nature 559, 73 (2018)

8. N.D. Hari Dass, Phys. Rev. Lett 36, 393 (1976)

9. L.S. Hou, W.T. Ni, Mod. Phys. Lett. A 16, 763 (2001)

10. Y.N. Obukhov, A.J. Silenko, O.V. Teryaev, Phys. Rev. D 90, 124068 (2014)

11. B.M. Barker, R.F. O’ Connell, Gen. Relativ. Gravit. 5, 539 (1974)

12. M. Mathisson, Acta Phys. Pol. 6, 163 (1937)

13. M. Mathisson, Acta Phys. Pol. 6, 218 (1937)

14. A. Papapetrou, Proc. R. Soc. A 209, 248 (1951)

15. B. Mashhoon, Ann. Phys. 89, 254 (1975)

16. R. Plyatsko, M. Fenyk, Phys. Rev. D 91, 064033 (2015)

17. W. Guzmán Ramrez, A.A. Deriglazov, Phys. Rev. D 92, 124017 (2015)

18. R.M. Plyatsko, O.B. Stefanyshyn, M.T. Fenyk, Class. Quantum Gravity 28, 195025 (2011)

19. O. Semerák, MNRAS 308, 863 (1999)

20. S.A. Hojman, F.A. Asenjo, Phys. Rev. D 93, 028501 (2016)

21. J. Steinhoff, Ann. Phys. (Berlin) 523, 296 (2011)

22. S.A. Hojman, F.A. Asenjo, Class. Quantum Gravity 34, 115011 (2017)

23. A.J. Hanson, T. Regge, Ann. Phys. 87, 498 (1974)

24. S.A. Hojman, Electromagnetic and Gravitational Interactions of a Spherical Relativistic Top. Ph.D. thesis, Princeton University (1975) (unpublished)

25. S. Hojman, T. Regge, in Studies in Mathematical Physics, Essays in Honor of Valentin Bargmann, ed. by E.H. Lieb, B. Simon and A.S. Wightman (Princeton University Press, Princeton, 1976), p. 195

26. R. Hojman, S. Hojman, Phys. Rev. D 15, 2724 (1977)

27. S. Hojman, Phys. Rev. D 18, 2741 (1978)
28. S.A. Hojman, F.A. Asenjo, Class. Quantum Gravity 30, 025008 (2013)

29. S.A. Hojman, B. Koch, Adv. High Energy Phys. 2013, Article ID 967805 (2013)

30. N. Zalaquett, S.A. Hojman, F.A. Asenjo, Class. Quantum Gravity 31, 085011 (2014). arXiv:1308.4435 [gr-qc]

31. C. Armaza, M. Bañados, B. Koch, Class. Quantum Gravity 33, $105014(2016)$

32. C. Armaza, S.A. Hojman, B. Koch, N. Zalaquett, Class. Quantum Gravity 33, 145011 (2016)

33. N. Zalaquett, Spinning Massive and Massless Test Particles in Curved Spacetime. Ph.D. Thesis, Pontificia Universidad Católica de Chile (2016) (unpublished)

34. Y.-P. Zhang et al., Phys. Rev. D 94, 124017 (2016)

35. E. Poisson, C.M. Will, Gravity: Newtonian, Post-Newtonian, Relativistic (Cambridge University Press, Cambridge, 2014)

36. B.M. Barker, R.F. O'Connell, Gen. Relativ. Gravit. 11, 149 (1979)

37. L.E. Kidder, C.M. Will, A.G. Wiseman, Phys. Rev. D 47, R4183 (1993)

38. L.E. Kidder, Phys. Rev. D 52, 821 (1995)

39. R.A. Porto, I.Z. Rothstein, Phys. Rev. D 78, 044013 (2008)

40. R.A. Porto, I.Z. Rothstein, Phys. Rev. Lett. 97, 021101 (2006)

41. J. Levin, Phys. Rev. Lett. 84, 3515 (2000)

42. M.D. Hartl, A. Buonanno, Phys. Rev. D 71, 024027 (2005)

43. J. Levin, Phys. Rev. D 74, 124027 (2006)

44. X. Wu, Y. Xie, Phys. Rev. D 76, 124004 (2007)

45. X. Wu, Y. Xie, Phys. Rev. D 77, 103012 (2008)

46. S.Y. Zhong, X. Wu, S.Q. Liu, X.F. Deng, Phys. Rev. D 82, 124040 (2010)

47. Y. Wang, X. Wu, Class. Quantum Gravity 28, 025010 (2011)

48. G. Huang, X. Ni, X. Wu, Eur. Phys. J. C 74, 3012 (2014)

49. L. Huang, X. Wu, D. Ma, Eur. Phys. J. C 76, 488 (2016)

50. J. Luo, X. Wu, G. Huang, F. Liu, ApJ 834, 64 (2017)

51. D. Bini, F. de Felice, A. Geralico, R.T. Jantzen, Class. Quantum Gravity 22, 2947 (2005)

52. S. Mukherjee, K.R. Nayak, (2018). arXiv:1804.06070

53. S. Suzuki, K.-I. Maeda, Phys. Rev. D 55, 4848 (1997)

54. C. Verhaaren, E.W. Hirschmann, Phys. Rev. D 81, 124034 (2010)

55. W. Tulczyjew, Acta Physica Polonica 18, 393 (1959)

56. G. d' Ambrosi, S. Satish Kumar, J. van de Vis, J.W. van Holten, Phys. Rev. D 93, 044051 (2016)

57. B.S. DeWitt, R.W. Brehme, Ann. Phys. 9, 220 (1960)

58. F.A. Asenjo, S.A. Hojman, Eur. Phys. J. C 77, 732 (2017)

59. F.A. Asenjo, S.A. Hojman, Class. Quantum Gravity 34, 205011 (2017)

60. F.A. Asenjo, S.A. Hojman, Phys. Rev. D 96, 044021 (2017)

61. J. Levin, Phys. Rev. D 67, 044013 (2003)

62. C. Königsdörffer, A. Gopakumar, Phys. Rev. D 71, 024039 (2005)

63. A. Gopakumar, C. Königsdörffer, Phys. Rev. D 72, 121501(R) (2005)

64. X. Wu, Y. Xie, Phys. Rev. D 81, 084045 (2010)

65. X. Wu, L. Mei, G. Huang, S. Liu, Phys. Rev. D 91, 024042 (2015)

66. X. Wu, G. Huang, MNRAS 452, 3167 (2015)

67. S.L. Shapiro, S.A. Teukolsky, Black Holes, White Dwarfs and Neutron Stars: The Physics of Compact Objects (Wiley, New York, 1983)

68. J.B. Hartle, Gravity: an introduction to Einstein's general relativity (Addison Wesley, Boston, 2003)

69. G. Schäfer, Gen. Relativ. Gravit. 36, 2223 (2004) 